\title{
Identification of proteins associated to multi-drug resistance in LoVo human colon cancer cells
}

\author{
MICHELE VISENTIN $^{1 *}$, MARIA PAOLA SIMULA ${ }^{1 *}$, FRANCA SARTOR $^{1}$, \\ ALESSANDRA PETRUCCO ${ }^{2}$, VALLI DE RE ${ }^{1}$ and GIUSEPPE TOFFOLI ${ }^{1}$ \\ ${ }^{1}$ Experimental and Clinical Pharmacology Unit, ${ }^{2}$ Experimental Oncology 2, CRO Centro di Riferimento \\ Oncologico, IRCCS National Cancer Institute, via Franco Gallini 2, 33081 Aviano (PN), Italy
}

Received October 21, 2008; Accepted December 12, 2008

DOI: 10.3892/ijo_00000255

\begin{abstract}
Multi-drug resistance (MDR) limits the effectiveness of chemotherapy. P-glycoprotein encoded by the MDRl gene, is known to be implicated in MDR phenotype, but other factors could be determinant in MDR. The aim of this study was to investigate new molecular factors potentially associated with the MDR phenotype using a proteomic approach. Two dimensional fluorescence difference gel electrophoresis (2D-DIGE) and MALDI-TOF peptide mass fingerprinting were used to determine differentially expressed proteins between LoVo human colon carcinoma cell line and one of its MDR sublines (LoVo-R1). Thirty-four differentially expressed proteins were identified. They were classified into five groups based on their biological functions: i) proteins involved in energy request pathways, ii) in detoxification pathways, iii) in cell survival activity, iv) in drug transport and v) in chaperone functions. Among these proteins, endothelin 1 and proteasome subunit $\beta 2$ regulations were validated by immunofluorescence and Western blotting, respectively, showing complete consistency with 2D-DIGE results. In conclusion, the proteomic approach indicates that multiple mechanisms are simultaneously involved in MDR. These might be useful in the search for new forms of interventional therapeutic approaches for MDR reversal.
\end{abstract}

Correspondence to: Dr Giuseppe Toffoli, Experimental and Clinical Pharmacology Unit, CRO Centro di Riferimento Oncologico, IRCCS National Cancer Institute, via Franco Gallini 2, 33081 AVIANO (PN), Italy

E-mail: gtoffoli@cro.it

*Contributed equally

Abbreviations: MDR, multi-drug resistance; 2D-DIGE, two dimensional difference in gel electrophoresis; MALDI-TOF, matrix-assisted laser desorption ionization-time-of-flight; P-gp, P-glycoprotein

Key words: multi-drug resistance, P-glycoprotein, difference in gel electrophoresis, mass spectrometry, proteomics

\section{Introduction}

Despite a significant progress in the pharmacological treatment of cancer, drug resistance remains the main factor limiting chemotherapy effectiveness. Tumors can be intrinsically resistant or may become resistant during chemotherapy treatment. Many factors affect drug sensitivity, including drug activation and inactivation, alterations in drug target, DNA methylation, alterations in DNA repair mechanisms, altered apoptosis and increased drug efflux. A frustrating property of chemoresistance is the common development of a characteristic phenotype named 'multi-drug resistance' (MDR), which is characterised by a simultaneous cross-resistance to many structurally and functionally unrelated drugs (1). MDR is generally due to the overexpression of P-glycoprotein (P-gp), the founding member of the ATP-binding cassette (ABC) proteins. These transporters share the ability to recognize and transport a large number of compounds, determining a reduced intracellular drug accumulation in an ATP-dependent manner $(2,3)$. Since the discovery of P-gp, a considerable research effort has been made to examine its role in resistance and for developing its function inhibitors. However, clinical studies on several chemosensitizers based on P-gp activity inhibition showed limited results, suggesting that further biochemical mechanisms, related or not to the increase in P-gp expression and/or activity, have a detrimental role in the MDR phenotype (4). On these grounds, the development of DNA microarrays and proteomic technologies can be useful to identify a panel of biomarkers that may be determinant for a better understanding of the underlying mechanisms of the MDR and could lead to the development of new chemosensitizers.

Conventional proteomics refers to the use of traditional two-dimensional gel electrophoresis (2-DE) for separating proteins, combined with mass spectrometry (MS) and database search to identify proteins. This comparative proteomic approach has just been successfully used to analyze laboratoryderived drug-resistant cancer cell lines and has allowed to discover novel mechanisms of resistance and to confirm already known ones (5).

The objective of this study was to identify proteins involved in MDR phenotype, documenting the expression changes between a human colon cancer chemosensitive cell line (LoVo) and the respective MDR subline (LoVo-R1), with a proteomic approach consisting in two dimensional fluorescence difference 
in gel electrophoresis (2D-DIGE) and protein identification combining peptide mass fingerprinting and matrix-assisted laser desorption ionization-time-of-flight mass spectrometry (MALDI-TOF MS).

\section{Materials and methods}

Materials. Doxorubicin (dox) was purchased from Ebewe Pharma (Vienna, Austria). 2D clean-up kit, Cyanine minimal labelling dyes Cy2 [3-(4-carboxymethyl)phenylmethyl)-3'ethyloxacarbocyanine halide $N$-hydroxysuccinimidyl ester], Cy3 [1-(5-carboxypentyl)-1'-propylindocarbocyanine halide $\mathrm{N}$-hydroxysuccinimidyl ester] and Cy5 [1-(5-carboxypentyl)1'-methylindodicarbocyanine halide $N$-hydroxysuccinimidyl ester] were obtained from GE Healthcare (Buckinghamshire, UK). Immobilized $\mathrm{pH}$ gradient buffer, immobilized $\mathrm{pH}$ gradient strips and precast gels were provided by Bio-Rad (Milan, Italy). The Peptide calibration Mix 4 was purchased from Laser Biolabs (Sophia-Antipolis, France). Mouse antihuman endothelin 1 and mouse anti-human proteasome subunit $\beta$ type 2 antibodies were purchased from SigmaAldrich (St. Louis, MO, USA) and from Santa Cruz Biotechnology (Santa Cruz, CA, USA), respectively.

Cell lines. The colon cancer cell line LoVo was purchased from American Culture Collection (Rockville, MD, USA). In our laboratory we established the LoVo drug-resistant sublines by single step dox selection. All the generated sublines have been found to be characterized by MDR phenotype. Among the chemoresistant subpopulations, the highest resistant one, named LoVo-R1, was selected for our experiments (2). LoVo and LoVo-R1 cell lines were harvested in F12 Ham's Nutrient (Biowhittaker, Europe). The medium was supplemented with $10 \%$ fetal bovine serum (Biowhittaker), streptomycin $10 \mu \mathrm{g} / \mathrm{ml}$ and penicillin $\mathrm{G} 10 \mathrm{U} / \mathrm{ml}$ (Biowhittaker). The MDR subpopulation was propagated in a medium containing $100 \mathrm{ng} / \mathrm{ml}$ dox. Cell cultures were incubated at $37^{\circ} \mathrm{C}$ in a humidified atmosphere of $5 \% \mathrm{CO}_{2}$ and $95 \%$ air.

Protein extraction. Cells were washed three times in a physiologic solution and then lysed (7 M urea, $2 \mathrm{M}$ thiourea, $100 \mathrm{mM}$ DTT, 4\% CHAPS e $40 \mathrm{mM}$ Tris $\mathrm{pH}$ 8.5). LoVo and LoVo-R1 protein lysates were precipitated according to the 2D clean-up kit protocol and then resuspended in $7 \mathrm{M}$ urea, $2 \mathrm{M}$ thiourea and 4\% CHAPS for 2D-electrophoresis. Protein concentration was determined by Bradford assay (Bio-Rad).

DIGE experimental design. Fluorescence 2D difference gel electrophoresis (DIGE) is commercialized as Ettan DIGE proteomics system (GE Healthcare). 2D-DIGE circumvents many of the issues associated with traditional 2D-PAGE as gel to gel variation and limited dynamic range and allows more accurate and sensitive quantitative proteomic studies. It is based on the specific properties of the three spectrally resolvable dyes: CyDye DIGE Fluor minimal dyes (Cy2, Cy3 and Cy5). 2D-DIGE technology allows to mix samples labelled with different cyanine dyes and resolve them on the same 2DE gel, affording an increase of the confidence associated with differences that are identified and quantified
Table I. Experimental design for minimal labelling two dimensional difference gel electrophoresis.

\begin{tabular}{lccc}
\hline Gel no. & Cy2 & Cy3 & Cy5 \\
\hline 1 & Pooled standard & LoVo-R1 & LoVo \\
2 & Pooled standard & LoVo-R1 & LoVo \\
3 & Pooled standard & LoVo & LoVo-R1 \\
4 & Pooled standard & LoVo & LoVo-R1 \\
\hline
\end{tabular}

Experimental design for the detection of differentially expressed proteins between the chemosensitive cell line LoVo and its MDR subline LoVo-R1. In our experimental model gels 1 and 2 are replicates of the same label scheme. Gels 3 and 4 derive from dye swapping strategy which is useful to avoid a dye labelling bias, observed at low spot volumes, owing to the different fluorescence characteristics of acrylamide at the different wavelengths of excitation for $\mathrm{Cy} 2, \mathrm{Cy} 3$ and $\mathrm{Cy} 5$ and to the different CyDye DIGE Fluors efficiencies in labelling different proteins. This multi-gel approach derived, allows further data points to be collected for each group to be compared.

among samples. Furthermore, the availability of three different dyes allows a common sample (pooled standard) to be run in all gels to aid in spot matching and normalization, thus reducing the rate of false negatives and false positives detected and providing a means to accurately identify even any modest change in protein levels. The internal standard is created by pooling aliquots from each individual sample in the experiment into one tube and corresponds to an equal amount of proteins from the samples being examined. The experimental design illustrated in Table I has been used for 2D-DIGE analysis. A dye-swapping scheme was used so that samples were never all labeled with $\mathrm{Cy} 3$ or $\mathrm{Cy} 5$ in order to prevent any dye-specific labeling artifact to occur.

$2 D$ gel electrophoresis and imaging. Twenty-five $\mu \mathrm{g}$ of individual samples and pooled internal standard, were minimallabelled with 100 pmol of Cy3/Cy5 and Cy2 respectively, following standard method. The labelled samples were then combined according to the experimental design (Table I), mixed with rehydration buffer (7 $\mathrm{M}$ urea, $2 \mathrm{M}$ thiourea, 4\% CHAPS, 40 mM DTT, 0.5\% immobilized pH gradient (IPG) buffer) and subjected to overnight passive rehydration on $11 \mathrm{~cm}$ immobilized $\mathrm{pH}$ gradient strips [IPG, $\mathrm{pH}$ 3-10 non linear (NL)]. Isoelectric focusing (IEF) was carried out on a Bio-Rad Protean IEF cell. The voltage program consisted of $15 \mathrm{~min}$ at $250 \mathrm{~V}$, a slow voltage ramp to $8000 \mathrm{~V}$ over $2-5 \mathrm{~h}$ and a final focusing step for a total of $35000 \mathrm{~V} / \mathrm{h}$. Focused IPG strips were stored at $-80^{\circ} \mathrm{C}$ before equilibration and application to SDS-PAGE.

For the second dimension, every strip was equilibrated (7 M urea, $2 \mathrm{M}$ thiourea, $2 \% \mathrm{SDS}, 30 \%$ glycerol, $50 \mathrm{mM}$ Tris- $\mathrm{HCl} \mathrm{pH} 8.8$ ) and proteins were reduced with $65 \mathrm{mM}$ DTT and alkylated with $135 \mathrm{mM}$ iodoacetamide. SDS-PAGE was run on Criterion IPG+1 Comb 8-16\% precast gels. Gels were scanned on a Typhoon Trio Scanner (GE Healthcare) at $100 \mu \mathrm{m}$ resolution in single scans applying each three wavelengths and filters (Cy2, Cy3 and Cy5). Gel images were 
subjected to Difference In-gel Analysis (DIA) and Biological Variation Analysis (BVA) using DeCyder version 6.5 software (GE Healthcare) which normalizes and statistically analyzes spots to identify and quantify differentially expressed proteins, allows matching of spots from multiple gels, calculates average abundance changes and statistically analyzes the differential protein expression. Spots with a value of $\mathrm{p}<0.05$ (Student's t-test) and an average change $>1.2$-fold were considered as proteins of interest.

Protein identification using MALDI-TOF mass spectrometry. Proteins of interest were picked from a colloidal Coomassiestained preparative gel, destained with $25 \mathrm{mM}$ ammonium bicarbonate in $50 \%$ acetonitrile and trypsin digested at $37^{\circ} \mathrm{C}$ overnight as previously reported (6). Peptides were then extracted with $1 \%$ trifluoroacetic acid (TFA) and subjected to a desalting and concentration step with Zip-Tip C18 (Millipore SPA, Milan, Italy). Peptide mass fingerprinting was performed on a Voyager-DE PRO Biospectrometry Workstation mass spectrometer (Applied Biosystems, Foster City, CA, USA). MALDI-TOF mass spectra were acquired in 700-4000 kDa molecular weight range, in positive ion mode, with $150 \mathrm{nsec}$ delay time and an ion acceleration voltage of $20 \mathrm{kV}$. Spectra were externally calibrated using Peptide calibration Mix 4 500-3500 kDa. Each spectrum, obtained by collecting 1000-2000 laser shots, was processed using Data Explorer version 5.1 software (Applied Biosystems). Database search was performed against two different non-redundant databases: NCBInr and Swiss-Prot, with the Mascot search engine (www.matrixscience.com) and the Aldente peptide mass fingerprinting tool (http://www.expasy.org/tools/ aldente), limiting the search to human proteins, allowing for one trypsin missed cleavage and with a 150 parts per million (p.p.m.) mass tolerance error.

Analysis of endothelin 1 expression by immunofluorescence. Cells were cultured on a $24 \times 24-\mathrm{mm}$ cover slip (Knittel Glaser, Germany) until confluence. The cells were washed, fixed in 4\% paraformaldehyde for $20 \mathrm{~min}$ and then soaked in PBS/BSA $1 \%$ containing $0.1 \%$ Triton X-100 and 2\% FBS, for 5 min. After three washes in PBS, they were blocked with PBS/BSA $1 \%$ containing $2 \% \mathrm{FBS}$ for $30 \mathrm{~min}$ at RT. The incubation with primary antibody was performed for $1 \mathrm{~h}$ at RT in the dark. For immunostaining, a mouse monoclonal antibody anti-endothelin 1 was used at a 1:200 working dilution in PBS/BSA $1 \%$. As secondary antibody we used a $\mathrm{F}\left(\mathrm{ab}^{\prime}\right)_{2}$ chicken anti-mouse IgG, Alexa-Fluor (AF) 488 conjugated (Invitrogen, CA, USA), diluted 1:200 in working solution (PBS/BSA 1\%). Nuclei were counterstained with a 1:5000 working solution of the nuclear stain ToPro-3 Iodide (Invitrogen). Fluorescent images were acquired with a Leica TCS SP2 confocal system (Leica Microsystems Heidelberg, Mannheim, Germany), using Leica confocal software. For the statistical evaluation of the data the paired Student's t-test was used.

Analysis of proteasome subunit $\beta$ type 2 expression by immunoblotting. Cells were washed three times in a physiological solution and suspended in lysis buffer (7 M urea, $2 \mathrm{M}$ thiourea, $100 \mathrm{mM}$ DTT, 4\% CHAPS e $40 \mathrm{mM}$ Tris
$\mathrm{pH}$ 8.5). Protein concentration was determined using a Bradford assay (Bio-Rad). Equal amounts of protein $(25 \mu \mathrm{g})$ were resolved on SDS-PAGE Criterion $4-12 \%$ precast gels (Bio-Rad) and transferred onto Protran Nitrocellulose Transfer membrane (Schleicher-Schuell, Dassel, Germany) using Trans Blot Semi-dry Transfer cell (Bio-Rad). The membranes were blocked overnight with $2 \%$ non-fat milk in Tris-buffered saline (TBS), $0.1 \%$ Tween-20 (TBS-T) and then incubated with anti-proteasome subunit $\beta$ type 2 primary antibody at 1:800 in blocking solution, followed by sheep anti-mouse IgG HRPlinked (GE Healthcare) at 1:1000 in blocking solution. Equal loading was confirmed using a goat anti-vinculin polyclonal antibody (clone N-19, Santa Cruz) at 1:1000, followed by a donkey anti-goat IgG-HRP antibody (Santa Cruz) as secondary reagent at 1:5000. Detection of immunoreactive proteins was accomplished with ECL Western blot analysis detection reagent (GE Healthcare) followed by autoradiography with Hyperfilm ECL (GE Healthcare). Films were scanned with a GS-800 densitometer (Bio-Rad). The densities of protein lanes were quantified with the densitometer's Quantity One software v.4.5 (Bio-Rad) to obtain an integral volume value (optical density, OD $x$ area). The expression of the proteasome subunit $\beta$ type 2 protein was normalized with respect to the vinculin. For the statistical evaluation of the data the paired Student's t-test was used.

\section{Results}

Analysis of differentially expressed proteins. After 2D-DIGE separation ( $\mathrm{pH}$ range 3.0-10.0), the Cy2, Cy3, and Cy5 channels of each gel were individually imaged to be then analyzed using DeCyder software. Sixty-six protein spots were significantly up-regulated (ratio LoVo-R1/LoVo $\geq 1.2$, $\mathrm{p} \leq 0.05$ ), whereas 84 were down-regulated in LoVo-R1 (ratio LoVo/LoVo-R1 $\geq 1.2, \mathrm{p} \leq 0.05$ ) compared to LoVo cell line. Fifty of the significant protein spots have been excised from the Coomassie stained preparative gel and subjected to MALDI-TOF peptide mass fingerprinting. The database search was performed against two different non-redundant databases: NCBInr and Swiss-Prot, with the Mascot search engine (www.matrixscience.com) and the Aldente peptide mass fingerprinting tool (http://www.expasy.org/tools/ aldente) for ensuring the reliability of the identification. Twenty-seven differentially expressed spots were successfully identified, by MALDI-TOF peptide mass fingerprinting. Fig. 1 shows the LoVo+LoVo-R1 pooled 2D image and the identified spots. The $\mathrm{p} I$ of the identified spots mostly ranged between 5.5 and 9 , and the molecular weight was in the range of $12-95 \mathrm{kDa}$. The database search revealed that 5 spots $(283,418,520,694$ and 869$)$ out of the 27 successfully identified were probably the result of the co-migration of two proteins, while spot no. 436, may include 3 different proteins. Therefore, the 27 identified spots represented 34 differentially expressed proteins. Sixteen (corresponding to 22 proteins) out of the 27 identified spots ( 34 proteins) were upregulated (Fig. 2) and 11 (12 proteins) were downregulated (Fig. 3) in the LoVo-R1 subline. The 34 differentially expressed proteins (comprehensive of the co-migrated proteins) were clustered into five groups according to their function: the proteins involved in i) energy request pathway, 


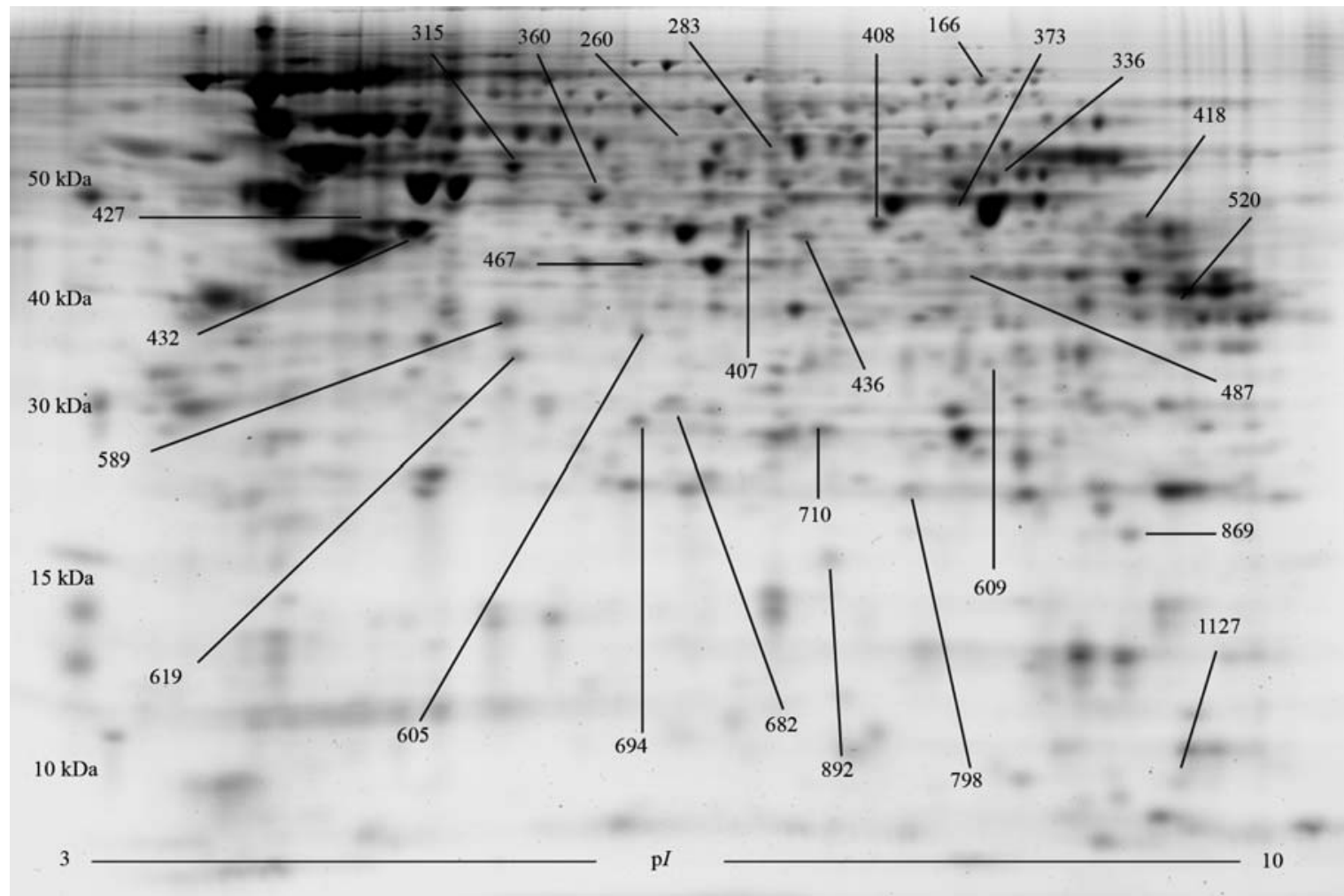

Figure 1. Two dimensional gel electrophoresis profile of LoVo and LoVo-R1 pool, pH range 3.0-10.0. For DIGE minimal labelling, Cy3 and Cy5 were used for sample labelling, while Cy2 labelled the pooled standard. Gels were scanned on a Thypoon Trio Scanner (100 $\mu \mathrm{m}$ resolution) in single scans applying three different wavelengths and filters each (Cy2, Cy3 and Cy5). The 2D pattern of a Cy2 labelled pooled standard is represented. The protein profiles were analyzed using DeCyder software 6.5. The annotations highlight the 27 spots (corresponding to 34 proteins) which were successfully identified by MALDITOF Peptide Mass fingerprinting.

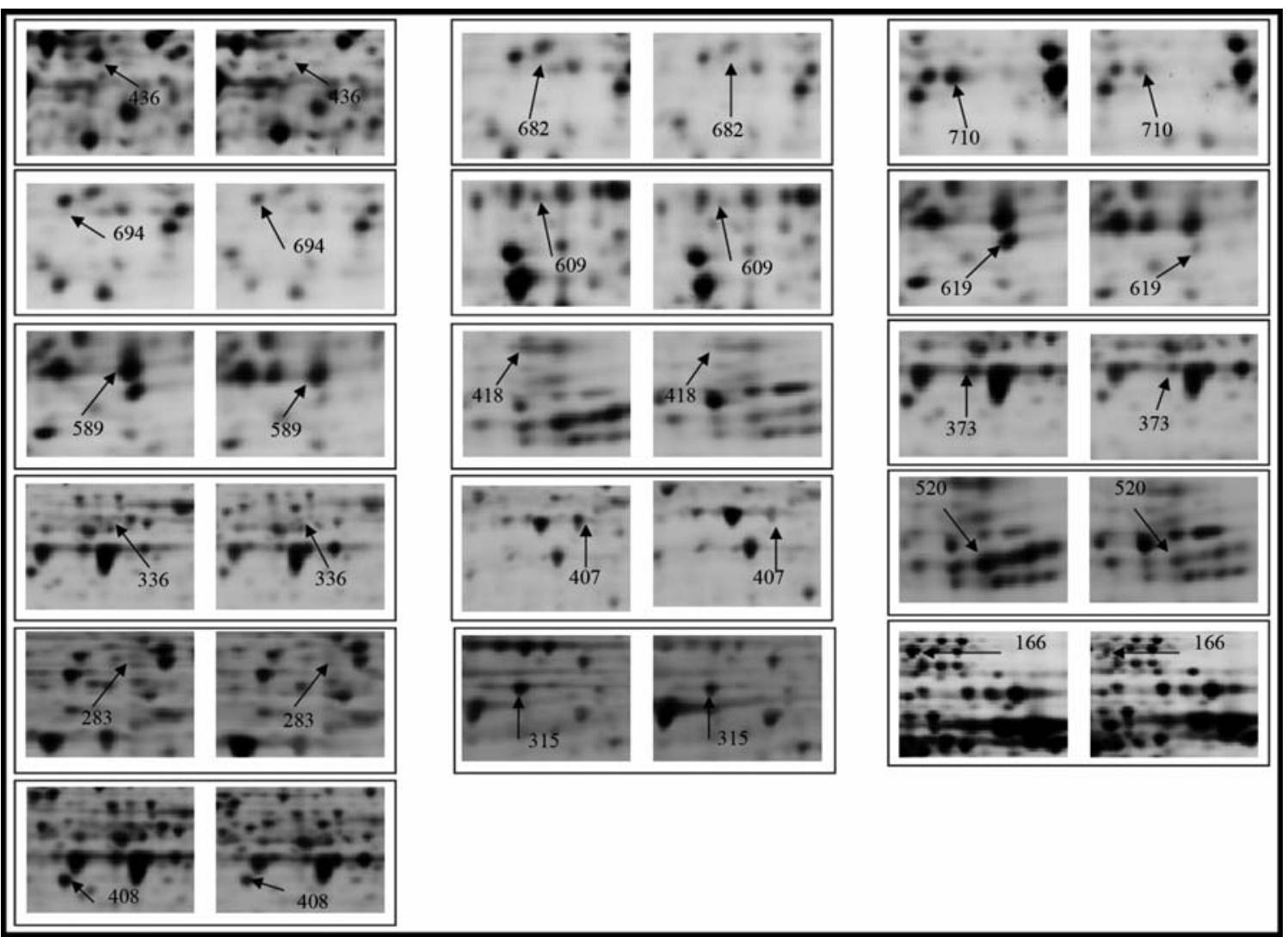

Figure 2. Identified spots upregulated in LoVo-R1 subline. DIGE gel maps of the 16 identified upregulated spots. Each panel shows the spot intensity in LoVo-R1 (left) and in LoVo (right) cells. In each panel, the spot of interest is highlighted with an arrow and identified with a specific number which refers to Table II. 


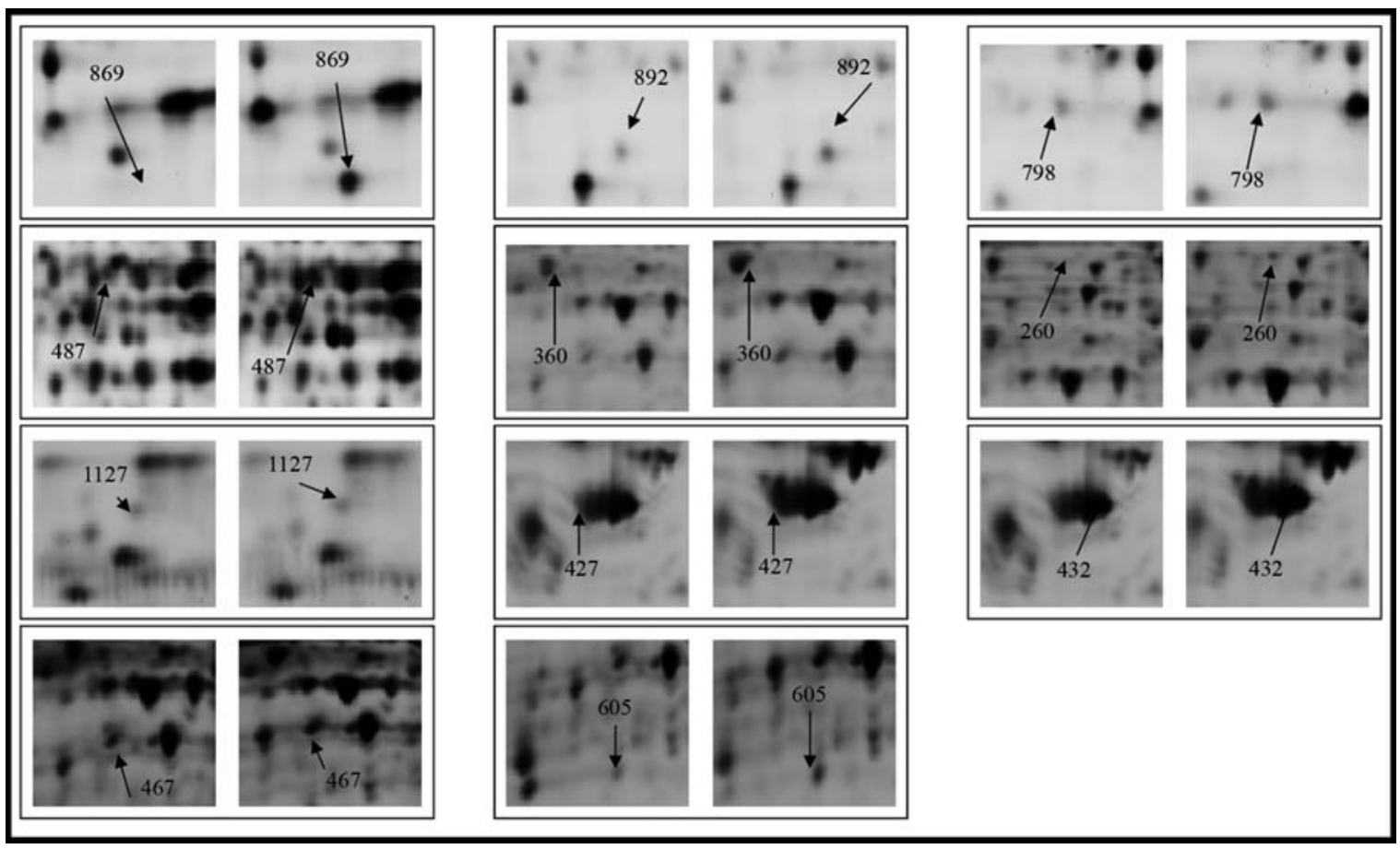

Figure 3. Identified spots downregulated in LoVo-R1 subline. DIGE gel maps of the 11 identified downregulated spots. As described for Fig. 2, each panel shows the spot intensity in LoVo-R1 (left) and in LoVo (right) cells. The spot of interest is highlighted with an arrow and identified with a specific number which refers to Table II.
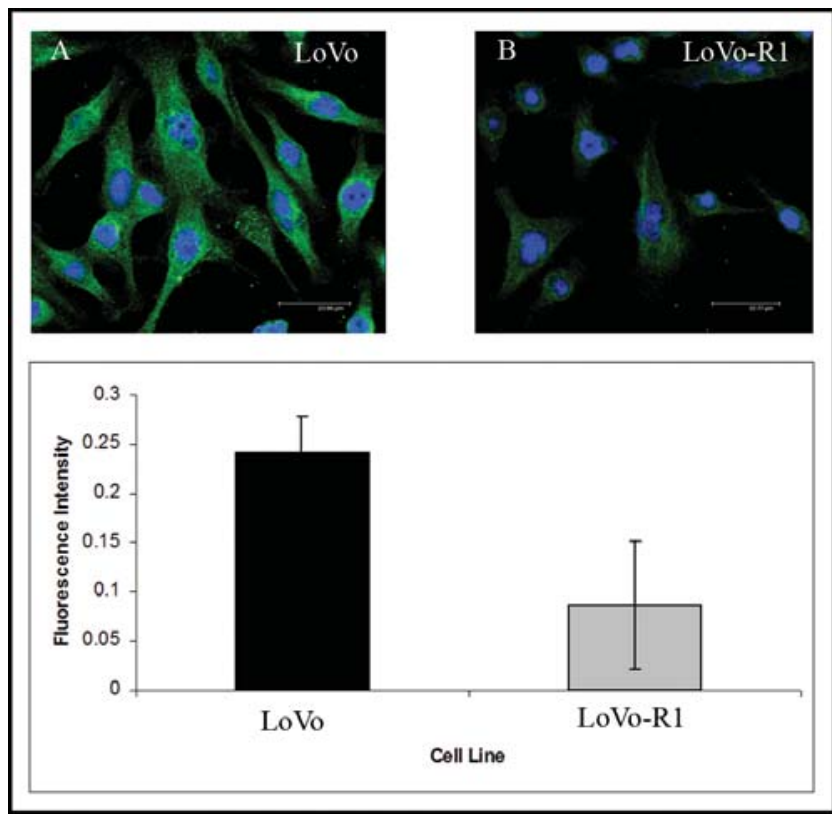

Figure 4. Pattern of ET-1 immunostaining in LoVo and in LoVo-R1 subline. Immunostaining that identifies ET-1-positive cells was performed using a mouse anti-ET-1 as primary and an Alexa-Fluor 488 conjugated anti-mouse $\mathrm{IgG}$ as secondary antibody. Nuclei were counterstained with the nuclear stain ToPro-3 iodide. Fluorescent images were obtained by a confocal laser scanning microscope, using a high resolution $63 \times 1.4$ numerical aperture oil immersion lens. Comparison of the two images reveals that ET-1 expression is more pronounced in sensitive LoVo cell line (A) while it is only moderately expressed in LoVo-R1 subline (B). To quantitatively evaluate the fluorescence intensity, representing ET-1 expression levels, Leica Confocal Software was used. In the histogram the relative fluorescence intensity is the mean \pm SD in LoVo and LoVo-R1 cells. The paired Student's t-test indicates that the ET-1 expression levels of LoVo and LoVo-R1 were significantly different $(\mathrm{p}=0.027)$. ii) in detoxification pathway, iii) in cell survival pathway, iv) in drug transport and v) in chaperone activities, defined as the ability of proteins to prevent RNA and protein misfolding. A comprehensive list of all the differentially expressed proteins identified, grouped by the biochemical pathway and/or activity, is reported in Table II.

Validation of differentially expressed proteins. Among the 34 differentially expressed proteins, proteasome 20S subunit $ß$ type 2 (PSMB2) and endothelin 1 (ET-1) were chosen for subsequent validation for their potential role in dox transport. Western blot analysis confirmed the downregulation of PSMB2 in LoVo-R1 while immunofluorescence assay was used to validate ET-1 modulation.

Fig. 4 represents the pattern of ET-1 immunostaining in LoVo and in LoVo-R1 subline. Comparison of the two images revealed that ET-1 expression was more pronounced in sensitive LoVo cell line while it was only moderately expressed in LoVo-R1 subline. The quantitative evaluation of the fluorescence intensity, representing ET-1 protein levels in LoVo and LoVo-R1 cells, performed using a Leica Confocal software, was reported (Fig. 4) as the mean \pm SD. The relative fluorescence intensity values were $0.24 \pm 0.036$ and $0.09 \pm 0.06$ in LoVo and LoVo-R1 cells respectively ( $\mathrm{p}=0.027)$.

Fig. 5 shows a representative Western blot analysis result of PSMB2 expression in LoVo and LoVo-R1. Immunoblot data confirmed that PSMB2 expression levels were lower in the resistant subline with respect to its sensitive counterpart. To quantitatively evaluate the densities of protein bands, representing PSMB2 expression levels, the densitometer's Quantity One software v.4.5 (Bio-Rad) was used. In the histogram (Fig. 5) we reported the lane intensity in LoVo and 


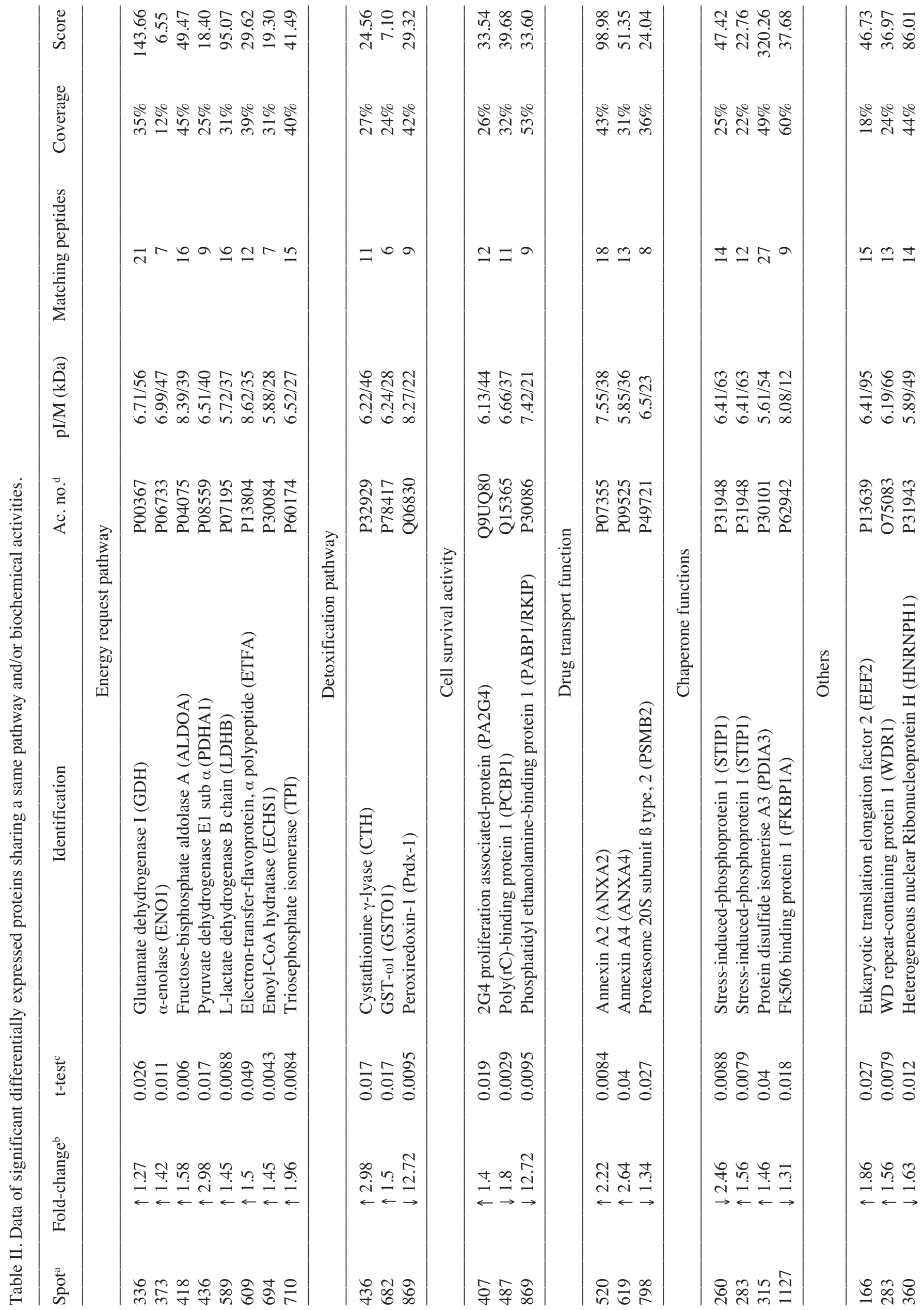



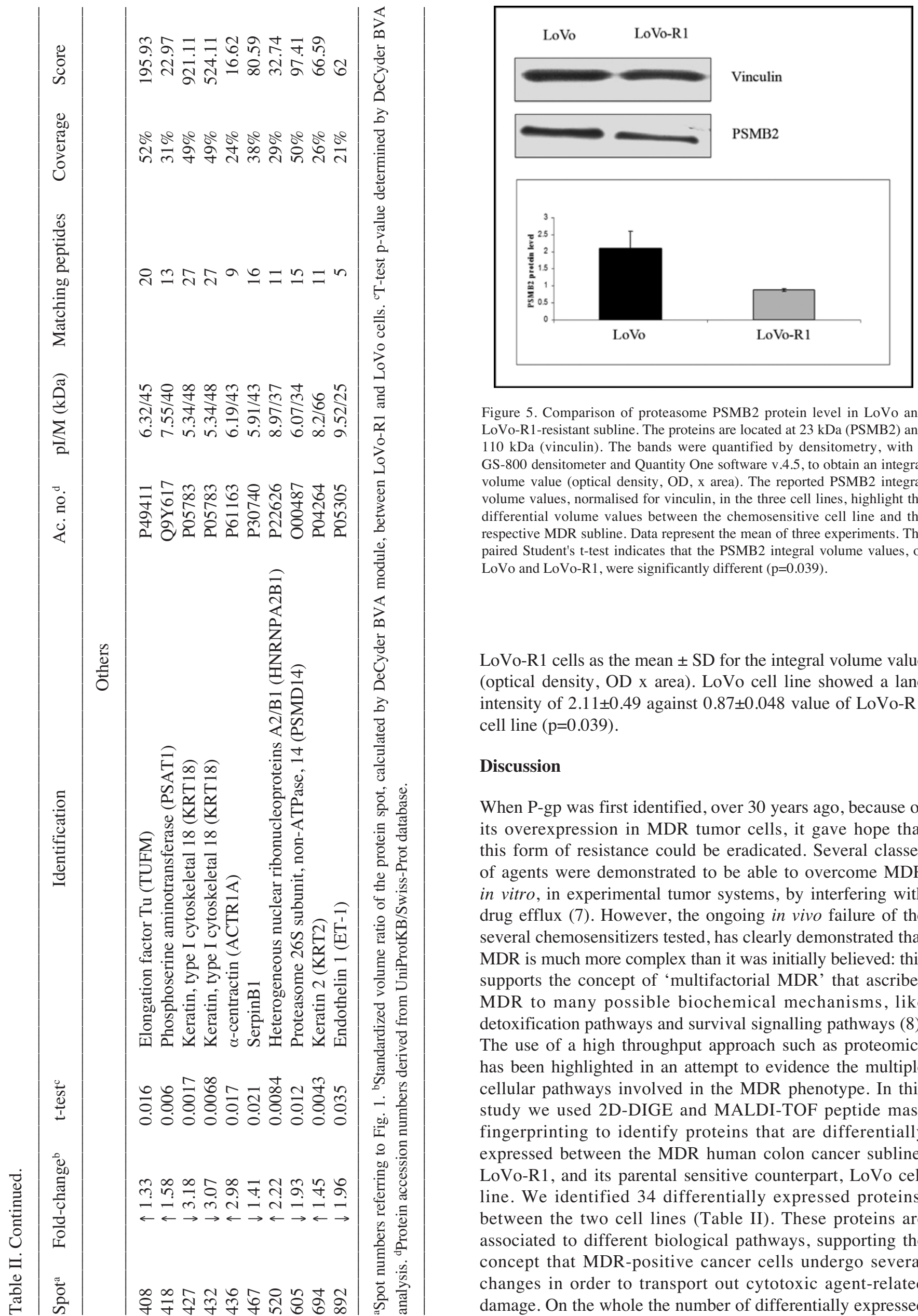

Figure 5. Comparison of proteasome PSMB2 protein level in LoVo and LoVo-R1-resistant subline. The proteins are located at $23 \mathrm{kDa}$ (PSMB2) and $110 \mathrm{kDa}$ (vinculin). The bands were quantified by densitometry, with a GS-800 densitometer and Quantity One software v.4.5, to obtain an integral volume value (optical density, OD, $x$ area). The reported PSMB2 integral volume values, normalised for vinculin, in the three cell lines, highlight the differential volume values between the chemosensitive cell line and the respective MDR subline. Data represent the mean of three experiments. The paired Student's t-test indicates that the PSMB2 integral volume values, of LoVo and LoVo-R1, were significantly different $(\mathrm{p}=0.039)$.

LoVo-R1 cells as the mean \pm SD for the integral volume value (optical density, OD $x$ area). LoVo cell line showed a lane intensity of $2.11 \pm 0.49$ against $0.87 \pm 0.048$ value of LoVo-R1 cell line $(\mathrm{p}=0.039)$.

\section{Discussion}

When P-gp was first identified, over 30 years ago, because of its overexpression in MDR tumor cells, it gave hope that this form of resistance could be eradicated. Several classes of agents were demonstrated to be able to overcome MDR in vitro, in experimental tumor systems, by interfering with drug efflux (7). However, the ongoing in vivo failure of the several chemosensitizers tested, has clearly demonstrated that MDR is much more complex than it was initially believed: this supports the concept of 'multifactorial MDR' that ascribes MDR to many possible biochemical mechanisms, like detoxification pathways and survival signalling pathways (8). The use of a high throughput approach such as proteomics has been highlighted in an attempt to evidence the multiple cellular pathways involved in the MDR phenotype. In this study we used 2D-DIGE and MALDI-TOF peptide mass fingerprinting to identify proteins that are differentially expressed between the MDR human colon cancer subline, LoVo-R1, and its parental sensitive counterpart, LoVo cell line. We identified 34 differentially expressed proteins, between the two cell lines (Table II). These proteins are associated to different biological pathways, supporting the concept that MDR-positive cancer cells undergo several changes in order to transport out cytotoxic agent-related damage. On the whole the number of differentially expressed 
proteins could be underestimated because 2D-PAGE approach poorly recovers large, small and hydrophobic proteins (9). In this context, as we expected, we did not find $\mathrm{ABC}$ transporter proteins among the differentially expressed ones, even if LoVo-R1 subline has been demonstrated to overexpress P-gp (2).

Some of the overexpressed proteins (8 out of 34 ) in LoVo-R1 are metabolic enzymes involved in generating energy. These enzymes are of particular importance in the maintenance of cell functionality since the disruption of metabolic pathways that normally support energy production, energy transfer and energy utilization directly contributes to cell injury. Previous studies indicate that glycolysis pathway components were increased in MDR neoplastic cells $(10,11)$, suggesting that the MDR cells enhance energy request. It must be considered that P-gp mediates an energy-dependent active drug efflux. In our study we found that the energyrelated protein triosephosphate isomerise (TPI) was increased $\sim 2$-fold, at the protein level, in LoVo-R1 cells $(+1.96)$. This glycolytic enzyme rapidly interconverts dihydroxyacetone phosphate with glyceraldehyde 3-phosphate, increasing glycolysis effectiveness and simplifying regulation. Another energy protein overexpressed in LoVo-R1 cells was the phosphoserine aminotransferase (PSAT) $(+1.58)$, an enzyme implicated in serine biosynthesis and also linked with cell proliferation in vitro (12). Three previous studies reported that PSAT1 mRNA is overexpressed in colon adenocarcinoma (13), showing an upregulation during tumor progression (14) and in colon chemoresistance (15). Our results support that PSAT1 overexpression is related to chemotherapy resistance.

Another group of differentially expressed proteins, in LoVo-R1 cells, is related to detoxification pathways. Glutathione-conjugation is the primary detoxification pathway and it is considered to be one of the major mechanisms of atypical MDR $(16,17)$. It is well known that anthracyclines act by generating iron-mediated free oxygen radicals that damage the DNA and cell membranes (18). The increase of intracellular reactive electrophiles stimulates the GST-GSH system and other thiolic factors that protect cellular macromolecules from reactive oxygen species (ROS) attack. We found GST- $\omega 1(+1.5)$ and cystathionine $\gamma$-lyase $(+2.98)$ upregulation in LoVo-R1 cells compared to LoVo cell line, suggesting a detoxification activity enhancement in order to contrast the radicals production deriving from dox exposure. Conversely, the antioxidant enzyme peroxiredoxin-1 (Prdx-1) was downregulated (-12.72) in LoVo-R1 subline. One of the most important biochemical effects of Prdx-1 is the inhibition

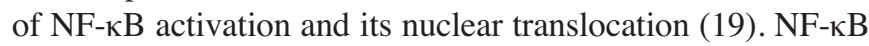
activation turns on the expression of genes that keep the cell proliferating and protects it from apoptosis (20). Thus, a reduction in Prdx-1 expression, and the consequent NF-кB activation, could favour LoVo-R1 survival signalling abrogating apoptosis in response to dox exposure.

It is now widely accepted that many chemotherapeutic agents simultaneously activate several different pathways that positively and negatively regulate the cell-death process, indicating that the balance between death and survival signal is critical in chemotherapy effectiveness (21). In this context survival pathways, that may have contributed to disease development in the first instance, could also be important in the development of the chemoresistance. Among the proteins involved in survival pathways we found a downregulation (-12.72) of the Raf kinase inhibitory protein (RKIP). RKIP is known as a modulator of the RAF/MAPK signalling cascade, a suppressor of metastasis and apoptosis $(20,22)$. On these grounds our results support previous data describing that elevated RKIP status may determine a positive clinical outcome probably by promoting chemotherapy-induced apoptosis (20).

The well-established idea that intracellular drug transport is a critical step for MDR phenotype is further supported, in our model, by the modulation of some proteins involved in carrier activity as Annexins A2 (+2.22), A4 (+2.64) and PSMB2 (-1.34). The modulation of these proteins indicates a cooperation with P-gp in the decrease of cellular/nuclear drug accumulation. Annexins A2 and A4 belong to a family of calcium- and phospholipid-binding proteins which may contribute to drug efflux through exocytosis of drug-filled vesicles (23). In this context, upregulation of these proteins could increase dox efflux in LoVo-R1 cells. On the contrary PSMB2 has been associated with anthracyclines nuclear transport. Indeed the proteasome seems to form a doxproteasome complex by binding of the drug to an allosteric site of the subunit with chymotrypsin-like activity (PSMB2). This complex translocates into the nucleus via nuclear pores and dissociates because of drug's higher affinity for DNA than for proteasome. The biochemical consequence of this interaction is the increased targeting of the chemotherapeutic agent at the nucleus compared to the passive diffusion only $(24,25)$. We found PSMB2 downregulated in LoVo-R1 cells compared to LoVo cells, suggesting that this protein may have a role in reducing dox nuclear uptake. These results seem to be in accordance with the previously observed lower dox nuclear accumulation in LoVo-R1 cells (26).

Finally, among the proteins classified as 'other functions', ET-1 (-1.96) deserves to be mentioned because of its role of modulator of MDRl gene expression and of P-gp activity. ET-1, a hormone primarily expressed in endothelial, vascular smooth muscle and epithelial cells (27), was demonstrated to be able to modulate P-gp and multi-drug resistance associated protein 2 (MRP-2) expression and activity. ET-1 pathway activation mediates the decreased cell-to-lumen transport of the fluorescent MRP2 substrate fluorescein methotrexate (FL-MTX) in killifish renal proximal tubule $(28,29)$. Moreover, Hartz et al found that ET-1, rapidly and reversibly, reduced P-gp-mediated transport at the blood-brain barrier in rat (30). On these grounds we suggest that ET-1 downregulation may play a role in the modulation of the MDR phenotype.

In short, our study supports that MDR development implies several adjustments by cancer cells, in order to prevent chemotherapeutic drug-induced damage, and provides further insights into the complex mechanisms of chemoresistance. In particular, our data highlight the role of proteins involved in energy request, detoxification and cell survival pathways, as well as the proteins directly involved in drug transport. This information may be useful as they could lead to the development of new functional tests in MDR investigation. Moreover, the in vivo confirmation of these data will be an important prerequisite to develop new forms of interventional therapeutic approaches in colon cancer. 


\section{Acknowledgements}

Authors wish to thank the 'Centro di Biomedicina Molecolare' (CBM), which provided the proteomic instruments, Dr Paola Spessotto for fluorescent image acquisition and Mrs. Anna Vallerugo for her writing assistance.

\section{References}

1. Longley DB and Johnston PG: Molecular mechanisms of drug resistance. J Pathol 205: 275-292, 2005.

2. Toffoli G, Viel A, Tumiotto L, Biscontin G, Rossi C and Boiocchi M: Pleiotropic-resistant phenotype is a multifactorial phenomenon in human colon carcinoma cell lines. Br J Cancer 63: 51-56, 1991 .

3. Ambudkar SV, Kim IW and Sauna ZE: The power of the pump: mechanisms of action of P-glycoprotein (ABCB1). Eur J Pharm Sci 27: 392-400, 2006.

4. Mcdevitt CA and Callaghan R: How can we best use structura information on P-glycoprotein to design inhibitors? Pharmaco Ther 113: 429-441, 2007

5. Zhang JT and Liu Y: Use of comparative proteomics to identify potential resistance mechanisms in cancer treatment. Cancer Treat Rev 33: 741-756, 2007.

6. De Re V, Simula MP, Cannizzaro R, Sansonno D, Canzonieri V, Gloghini A, Carbone A, Colombatti A, Marin MD, De Zorzi M and Toffoli G: HCV inhibits antigen processing and presentation and induces oxidative stress response in gastric mucosa. Proteomics Clin Appl 2: 1290-1299, 2008.

7. Toffoli G, Corona G, Sorio R, Bertola A and Boiocchi M: Reversal activity of cyclosporin A and its metabolites M1, M17 and M21 in multidrug-resistant cells. Int J Cancer 71: 900-906, 1997.

8. Wilson TR, Longley DB and Johnston PG: Chemoresistance in solid tumours. Ann Oncol 17 (Suppl 10): x315-x324, 2006.

9. Rahbar AM and Fenselau C: Unbiased examination of changes in plasma membrane proteins in drug resistant cancer cells. Proteome Res 4: 2148-2153, 2005

10. Chen G, Gharib TG, Wang H, Huang CC, Kuick R, Thomas DG, Shedden KA, Misek DE, Taylor JM, Giordano TJ, Kardia SL, Iannettoni MD, Yee J, Hogg PJ, Orringer MB, Hanash SM and Beer DG: Protein profiles associated with survival in lung adenocarcinoma. Proc Natl Acad Sci USA 100: 13537-13542. 2003.

11. Chuthapisith S, Layfield R, Kerr ID, Hughes C and Eremin O: Proteomic profiling of MCF-7 breast cancer cells with chemoresistance to different types of anti-cancer drugs. Int $\mathbf{J}$ Oncol 30: 1545-1551, 2007.

12. Baek JY, Jun DY, Taub D and Kim YH: Characterization of human phosphoserine aminotransferase involved in the phosphorylated pathway of L-serine biosynthesis. Biochem J 373: 191-200, 2003

13. Friederichs J, Rosenber GR, Mages J, Janssen KP, Maeckl C, Nekarda H, Holzmann B and Siewert JR: Gene expression profiles of different clinical stages of colorectal carcinoma: toward a molecular genetic understanding of tumor progression. Int J Colorectal Dis 20: 391-402, 2005.
14. Ojala P, Sundstrom J, Gronroos JM, Virtanen E, Talvinen K and Nevalainen TJ: mRNA differential display of gene expression in colonic carcinoma. Electrophoresis 23: 1667-1676, 2002.

15. Vie N, Copois V, Bascoul-Mollevi C, Denis V, Bec N, Robert B, Fraslon C, Conseiller E, Molina F, Larroque C, Martineau P, Del Rio $\mathrm{M}$ and Gongora $\mathrm{C}$ : Overexpression of phosphoserine aminotransferase PSAT1 stimulates cell growth and increases chemoresistance of colon cancer cells. Mol Cancer 7: 14, 2008.

16. Zhang D and Fan D: Multidrug resistance in gastric cancer: recent research advances and ongoing therapeutic challenges. Expert Rev Anticancer Ther 7: 1369-1378, 2007.

17. Stavrovskaya AA: Cellular mechanisms of multidrug resistance of tumor cells. Biochemistry (Mosc) 65: 95-106, 2000.

18. Minotti G, Menna P, Salvatorelli E, Cairo G and Gianni L: Anthracyclines: molecular advances and pharmacologic developments in antitumor activity and cardiotoxicity. Pharmacol Rev 56: 185-229, 2004

19. Hansen JM, Moriarty-Craige S and Jones DP: Nuclear and cytoplasmic peroxiredoxin-1 differentially regulate NF-kappaB activities. Free Radic Biol Med 43: 282-288, 2007.

20. Odabaei G, Chatterjee D, Jazirehi AR, Goodglick L, Yeung K and Bonavida B: Raf-1 kinase inhibitor protein: structure, function, regulation of cell signaling, and pivotal role in apoptosis. Adv Cancer Res 91: 169-200, 2004.

21. Nakanishi $\mathrm{C}$ and Toi M: Nuclear factor-kappaB inhibitors as sensitizers to anticancer drugs. Nat Rev Cancer 5: 297-309, 2005.

22. Trakul N, Menard RE, Schade GR, Qian Z and Rosner MR: Raf kinase inhibitory protein regulates Raf-1 but not B-Raf kinase activation. J Biol Chem 280: 24931-24940, 2005.

23. Wang Y, Serfass L, Roy MO, Wong J, Bonneau AM and Georges E: Annexin-I expression modulates drug resistance in tumor cells. Biochem Biophys Res Commun 314: 565-570, 2004.

24. Kiyomiya K, Matsuo S and Kurebe M: Mechanism of specific nuclear transport of adriamycin: the mode of nuclear translocation of adriamycin-proteasome complex. Cancer Res 61: 2467-2471, 2001.

25. Kiyomiya K, Matsuo $\mathrm{S}$ and Kurebe M: Proteasome is a carrier to translocate doxorubicin from cytoplasm into nucleus. Life Sci 62: 1853-1860, 1998 .

26. Boiocchi $\mathrm{M}$ and Toffoli G: Mechanism of multidrug resistance in human tumour cell lines and complete reversion of cellular resistance. Eur J Cancer 28A: 1099-1105, 1992.

27. Wulfing P, Tio J, Kersting C, Sonntag B, Buerger H, Wulfing C, Euler U, Boecker W, Tulusan AH and Kiesel L: Expression of endothelin-A-receptor predicts unfavourable response to neoadjuvant chemotherapy in locally advanced breast cancer. $\mathrm{Br}$ J Cancer 91: 434-440, 2004.

28. Notenboom S, Miller DS, Smits P, Russel FG and Masereeuw R: Role of NO in endothelin-regulated drug transport in the renal proximal tubule. Am J Physiol Renal Physiol 282: F458-F464, 2002.

29. Masereeuw R, Terlouw SA, Van Aubel RA, Russel FG and Miller DS: Endothelin B receptor-mediated regulation of ATPdriven drug secretion in renal proximal tubule. Mol Pharmacol 57: 59-67, 2000.

30. Hartz AM, Bauer B, Fricker G and Miller DS: Rapid regulation of P-glycoprotein at the blood-brain barrier by endothelin-1. Mol Pharmacol 66: 387-394, 2004 